\title{
Cardiac Arrhythmias in COVID-19
}

\author{
Małgorzata Mierzejewska,A-D, Marcin Grabowski ${ }^{1, \mathrm{~A}, \mathrm{E}-\mathrm{F}}$ \\ A - Research concept and design, B - Collection and/or assembly of data, C - Data analysis and interpretation, \\ D - Writing the article, E - Critical revision of the article, F - Final approval of article
}

$11^{\text {st }}$ Department of Cardiology, Medical University of Warsaw

Address for correspondence:

Dr. Małgorzata Mierzejewska, $1^{\text {st }}$ Department of Cardiology, Medical University of Warsaw email: mmierzejewska97@gmail.com

Prof. Marcin Grabowski, $1^{\text {st }}$ Department of Cardiology, Medical University of Warsaw email: marcin.grabowski@wum.edu.pl

Received: 2020-07-20

Revised:

Accepted: 2020-11-30

Final review:

DOI:

\section{Key words:}

\section{COVID-19, coronavirus, arrhythmia}

\begin{abstract}
Although the novel coronavirus, specifically known as severe acute respiratory syndrome coronavirus 2 (SARS-CoV-2), was thought to primarily affect the respiratory system, with the new studies and cases it seems that an effect on the cardiovascular system may also be prevalent. The manifestations include myocardial injury, exhibited by elevated TnT levels, as well as elevated levels of NT-proBNP and creatine kinase
\end{abstract}

\section{Introduction}

The most common non-specific COVID-19 symptoms include fever, cough and shortness of breath, which may progress to severe pneumonia or acute respiratory distress syndrome (ARDS) with prominent and characteristic abnormalities in radiological examination. Patients already suffering from diabetes, hypertension and other cardiovascular diseases such as coronary heart disease or cardiomyopathy have been reported to suffer from a more severe presentation of COVID-19. ${ }^{[1]}$ Coronavirus disease was reported to also manifest in the cardiovascular system, and its manifestation can be due to already present and now more severe cardiomyopathy or coronary heart disease. In $44, .4 \%$ of intensive care unit patients cardiac arrhythmias were reported, although the characteristics of those associated with COVID-19 have not yet been clearly described. In this article we focus on cardiac arrhythmias in COVID-19, which have been associated with underlying myocardial injury and cardiac dysfunction.

exceptionally cardiotropic without any involvement of the respiratory system. ${ }^{[2]}$

\section{Cardiovascular manifestations of COVID-19}

Regarding the involvement of the cardiovascular system, various manifestations have been reported, including myocardial injury indicated by elevated TnT levels, hypotension, hypoxia, as well as cardiac arrhythmias, to name a few. ${ }^{[3][4][5]}$

Elevated levels of NT-proBNP and creatine kinase were also noticeable due to an exceptionally cardiotropic manifes- 
tation of COVID-19 or an already present cardiomyopathy or coronary heart disease intensified by the viral infection. ${ }^{[4]}$

The case report by Inciardi et al. ${ }^{[2]}$ described an otherwise healthy 53-year-old woman who presented to the emergency department with severe fatigue, fever and dry cough for the past week. While chest radiography was unremarkable and there was no evidence of obstructive coronary heart disease, the patient developed acute myocardial inflammation without prominent involvement of the respiratory system, such as interstitial pneumonia, in this presentation of COVID-19.

\section{Cardiac arrhythmias}

The evidence of cardiac arrhythmias in COVID-19 patients has not been clearly described - Rajesh et al ${ }^{[6]}$ stated that arrhythmias were seen in $16.7 \%$ of patients, while Wang et al. ${ }^{[7]}$ reported that $44.4 \%$ of intensive care unit individuals had experienced cardiac arrhythmias. Chen et al. ${ }^{[8]}$ observed sinus tachycardia in 23 severe patients and all the critical patients, which made it the most common type of arrhythmia. At the end stage in critical patients both ventricular tachycardia and atrioventricular block were observed, while atrial fibrillation and bradycardia were less common types of arrhythmias.

Wu et al. ${ }^{[9]}$ stated that in $17 \%$ of 138 hospitalized patients with COVID-19 no further specified arrhythmias were reported, and additional attention is recommended in treating patients with distinct inherited arrhythmia syndromes, who may be susceptible to proarrhythmic effects of COVID-19 those inherited syndromes seem to be of no less importance than concomitant comorbidities affecting the progress of the infection. Management includes electrocardiographic monitoring, as well as intensified antipyretic pharmacotherapy in case of an arrhythmia-inducing fever or stress. Electrolyte abnormalities and certain antiviral medication also have an effect on the heart's rhythm - chloroquine and hydroxychloroquine, which can potentially be used in patients with COVID-19, present an adverse effect by lengthening the QT interval, but as the therapy lasts 5 to 10 days the risk seems to be relatively low. ${ }^{[10]}$ Naturally, in patients with congenital short QT syndromes this type of effect might be beneficial.

A study by Kochav et al. ${ }^{[3]}$ presented 4 cases of possible manifestations of COVID-19 which included atrioventricular block, atrial fibrillation, polymorphic ventricular tachycardia and cardiogenic shock. Although there seemed to be no direct correlation between the occurrence of cardiac arrhythmias and the severity of lung injury in COVID-19 patients, atrial fibrillation in critically ill patients has been associated with poor outcomes, and in the case of a fulminant myocarditis a rapid hemodynamic decline can be demonstrated.

\section{Management of arrhythmias}

As SARS-CoV-2 has been reported to spread through person-to-person transmission, standard precautions should be adhered to - in the case of an indication for catheter ablation a case-by-case approach is recommended, as well as possible postponement of such procedures except for life-threatening conditions. Also, postponing implantation of antiarrhythmic devices in stable patients and same day discharge after implantation of the device should both be considered to minimize the risk of COVID-19 infection. ${ }^{[1]}$

Pharmacotherapy of life-threatening cardiac arrhythmias includes betablockers as first-line therapy in both patients with atrial fibrillation or flutter and ventricular tachycardia or fibrillation. Amiodarone may be used in patients with VT/VF as a first-line agent, but it must be administered with caution - its potential QT-prolonging adverse effect must be monitored, especially when chloroquine, hydroxychloroquine or macrolides are also in use. Other methods of minimizing the risk of arrhythmia are frequent monitoring of electrolytes as well as possible avoidance of oral anticoagulants when immunomodulatory or antiviral treatment are in use due to possible drug interactions..$^{[1]}$

The use of external defibrillation/cardioversion has also been described in management of arrhythmias, although they should be limited in use to conditions such as supraventricular tachycardia or refractory cases of $\mathrm{AF} /$ atrial flutter which are not responding to other types of therapy - in stable patients cardioversion should be postponed. ${ }^{[10[111]}$

\section{Conclusions}

Cardiologists are faced with new challenges as COVID-19 seems to also affect the cardiovascular system, with arrhythmias being a much more common manifestation than was initially expected.

The proposed therapy for coronavirus-disease includes potential QT-prolonging drugs such as chloroquine and hydroxychloroquine, which can exacerbate the possible pro-arrhythmic effect - therefore additional and frequent electrolyte and ECG monitoring is recommended, even if the antiviral therapy seems to be relatively short, lasting 5 to 10 days. ${ }^{[10[111]}$

Practicing social distancing, frequent handwashing and self-isolation remain the most efficient ways to minimize the risk of infection, with consultations and monitoring done without face-to-face contact if possible. ${ }^{[11]}$

To achieve further and better understanding of how the virus spreads and how it affects various systems, future studies are needed - on treating the cardiovascular manifestations and arrhythmias associated with the infection, as well as on other manifestations of SARS-CoV-2, which may be reported as more information is gained by the end of the pandemic.

\section{References}

1. Li B, Yang J, Zhao F, Zhi L, Wang X, Liu L, Bi Z, Zhao Y. Prevalence and impact of cardiovascular metabolic 
diseases on COVID-19 in China. Clinical Research in Cardiology doi: 10.1007/s00392-020-01626-9

2. Inciardi RM, Lupi L, Zaccone G, Italia L, Raffo M, Tomasoni D, Cani DS, Cerini M, Farina D, Gavazzi E, Maroldi R, Adamo M, Ammirati E, Sinagra G, Lombardi CM, Metra M. Cardiac Involvement in a Patient With Coronavirus Disease 2019 (COVID-19). JAMA Cardiol. March 27, 2020. doi:10.1001/jamacardio.2020.1096

3. Kochav SM, Coromilas E, Nalbandian A, Ranard LS, Gupta A, Chung MK, Gopinathannair R, Biviano AB, Garan H, Wan EY. Cardiac Arrhythmias in COVID-19 Infection. Circ Arrhythm Electrophysiol. 2020;13:e008719. doi: 10.1161/CIRCEP.120.008719

4. Guo T, Fan Y, Chen M, Wu X, Zhang L, He T, Wang H, Wan J, Wang X, Lu Z. Cardiovascular Implications of Fatal Outcomes of Patients With Coronavirus Disease 2019 (COVID-19). JAMA Cardiol. 2020 Mar 27. doi: 10.1001/jamacardio.2020.1017

5. Kochi AN, Tagliari AP, Forleo GB, Fassini GM, Tondo C. Cardiac and Arrhythmic Complications in Patients With COVID-19. J Cardiovasc Electrophysiol. 2020 May;31(5):1003-1008. doi: 10.1111/jce.14479

6. Rajan R, Jarallah MAI, Dashti R. Cardiovascular complications of novel Wuhan Coronavirus (COVID-19) -A 2020 update. J Cardiol Curr Res. 2020;13(1):28. doi: 10.15406/jccr.2020.13.00468

7. Wang $\mathrm{D}, \mathrm{Hu} \mathrm{B}, \mathrm{Hu} \mathrm{C}$, Zhu F, Liu $X$, Zhang J, Wang B, Xiang H, Cheng Z, Xiong Y, Zhao Y, Li Y, Wang $X$, Peng Z. Clinical characteristics of 138 hospitalized patients with 2019 novel coronavirus-infected pneumonia in Wuhan, China. JAMA. 2020;323:1061-1069. doi: 10.1001/jama.2020.1585

8. Chen Q, Xu L, Dai Y, Ling Y, Mao J, Qian J, Zhu W, Di W, Ge J. Cardiovascular manifestations in severe and critical patients with COVID-19. Clin Cardiol. 2020;1-7. doi: 10.1002/clc.23384

9. Wu CI, Postema PG, Arbelo E, Behr ER, Bezzina CR, Napolitano C, Robyns T, Probst V, Schulze-Bahr E, Remme CA, Wilde AAM. SARS-CoV-2, COVID-19, and inherited arrhythmia syndromes. Heart Rhythm 2020;-:1-7. doi: 10.1016/j.hrthm.2020.03.024

10. Kuck KH. Arrhythmias and sudden cardiac death in the COVID-19 pandemic. Herz. 2020 Apr 24 : 1-2. doi: 10.1007/s00059-020-04924-0

11. Saenz LC, Miranda A, Speranza R, Alkmim R, Rojel T, Rojel U, Enriquez E, Figuereido M.
12. Recommendations for the organization of electrophysiology and cardiac pacing services during the COVID-19 pandemic. Latin American Heart Rhythm Society (LAHRS) in collaboration with: Colombian College Of Electrophysiology, Argentinian Society of Cardiac Electrophysiology (SADEC), Brazilian Society Of Cardiac Arrhythmias (SOBRAC), Mexican Society Of Cardiac Electrophysiology (SOMEEC). Journal of Interventional Cardiac Electrophysiology. doi:10.1007/ s10840-020-00747-5 